\title{
Roles of YAP in mediating endothelial cell junctional stability and vascular remodeling
}

\author{
Hyun-Jung Choi ${ }^{1}$ E Young-Guen Kwon ${ }^{2}$ * \\ ${ }^{1}$ Severance Integrative Research Institute for Cerebral \& Cardiovascular Diseases (SIRIC), College of Medicine, Yonsei University, \\ ${ }^{2}$ Department of Biochemistry, College of Life Science and Biotechnology, Yonsei University, Seoul 03722, Korea
}

\begin{abstract}
Angiogenesis is a complex process involving dynamic interaction of various cell to cell interactions. Endothelial cell interactions regulated by growth factors, inflammatory cytokines, or hemodynamic stress are critical for balancing vascular quiescence and activation. Yes-associated protein (YAP), an effector of Hippo signaling, is known to play significant roles in maintaining cellular homeostasis. However, its role in endothelial cells for angiogenic regulation remains relatively unexplored. We demonstrated the critical role of YAP in vascular endothelial cells and elucidated the underlying molecular mechanisms involved in angiogenic regulation of YAP. YAP was expressed in active angiogenic regions where endothelial cell junctions were relatively loosened. Consistently, YAP subcellular localization and activity were regulated by VE-cadherin-mediated PI3K/Akt pathway. YAP thereby regulated endothelial sprouting via angiopoietin-2 expression. These results provide an insight into a model of coordinating endothelial junctional stability and angiogenic activation through YAP. [BMB Reports 2015; 48(8): 429-430]
\end{abstract}

Yes-associated protein (YAP), a transcriptional co-activator, has been implicated in contact inhibition of cell growth and control of organ size. YAP activity is regulated in part by phosphorylation and subcellular localization through Hippo pathway. Recently, YAP has been suggested to play a role in mechanotransduction in Hippo-independent manner. Given that

*Corresponding author. E-mail: ygkwon@yonsei.ac.kr

http://dx.doi.org/10.5483/BMBRep.2015.48.8.146

Received 9 July 2015

Keywords: YAP, ANG-2, VE-cadherin, Akt, Endothelial junction, Angiogenesis

Abbreviations: YAP, Yes-associated protein; ANG-2, angiopoietin-2; VE-cadherin, vascular-endothelial cadherin; HUVEC, human umbilical vein endothelial cell

Perspective to: Hyun-Jung Choi et al (2015), Yes-associated protein regulates endothelial cell contact-mediated expression of angiopoietin-2, Nature Communications, May 12;6:6943. doi: 10.1038/ ncomms 7943 endothelial cell-cell contact and hemodynamic force is critical for the regulation of vascular homeostasis, YAP is expected to play important roles in endothelial cell function. A previous study showing the defects in yolk sac vasculogenesis of Yap knockout mice (E.M. Morin-Kensicki et al. Mol Cell Biol 26: 77-87. doi: 10.1128) also supports this hypothesis. YAP has also been reported to regulate endothelial cell proliferation in vitro (Z. Shen et al. PloS One, 2015;10(1); e0117522. doi: 10.1371). Nonetheless, the expression and functions of YAP in endothelial cells of vessels are not clearly determined yet. Our study has shown that YAP is expressed in developing mouse retinal vessels and tumor vessels. Subcellular localization and nuclear activity of YAP in endothelial cells was regulated by cell to cell contact similar to other cell types. Suppression of YAP in endothelial cells inhibited endothelial cell function such as the ability of forming tubular network on Matrigel or sprouting from EC-coated microbeads. Further, endothelial sprouting from mouse aortic ring and vascular branching of mouse retinal vessel was also affected by YAP depletion.

New vessel formation derived from pre-existing vessels is called angiogenesis. It occurs through activation of quiescent endothelial cells upon stimulation. Endothelial activation is accompanied by instability of cell to cell junction. VE-cadherin, an endothelial specific junctional molecule, not only mediates adherence junctional formation, but also triggers intracellular signaling for cell dynamics and proliferation. Several transcriptional factors including FoxO1 and $\beta$-catenin mediates VE-cadherin-induced signaling in genetic regulation. We identified YAP as an important transcriptional regulator that transduces signal from VE-cadherin mediated endothelial cell to cell contact to the nucleus. Our current study showed that in quiescent endothelial cells, VE-cadherin expression and clustering was important in maintaining the inactivated of YAP through phosphorylation. Disruption of VE-cadherin clustering or suppression of VE-cadherin expression resulted in the activation of YAP. We also showed that VE-cadherin dependent YAP phosphorylation required the activation of PI3K/Akt signaling pathway. Nuclear localization and transcriptional activity of YAP was increased by genetic or pharmacologic blockade of Akt pathway in confluent endothelial cells.

Angiopoietin-2 (ANG-2), an autocrine factor released from active endothelial cells, plays important role in vascular re- 


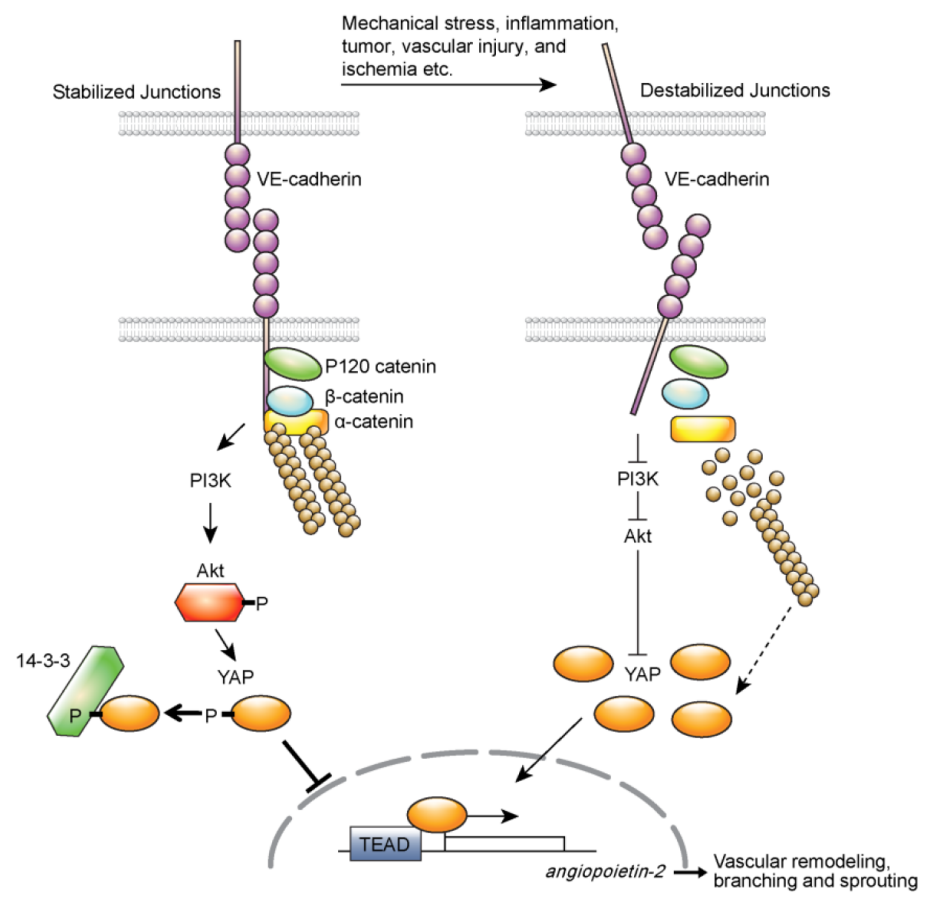

Fig. 1. YAP may be maintained in inactivated state by VE-cadherin-PI3/Akt signaling axis in quiescent endothelial cells. VE-cadherin clustering not only induces catenine complex formation, but also induces Akt activation pathways. When VE-cadherin-mediated cell to cell contact is disrupted in a variety of pathophysiological conditions, PI3/Akt pathway is inactivated, which results in nuclear localization of YAP. Nuclear YAP induces ANG-2 for angiogenic sprouting and vascular remodeling. Destabilized actin cytoskeleton might also affect YAP activity in endothelial cells. This requires further investigations (Figure is reproduced from an original article published in Nature Communications).

modeling. Although it is well known that ANG-2 synthesis and secretion are dynamically regulated in endothelial cells, detailed mechanism of its regulation is not clearly understood. Here, we identified ANG-2 as a transcriptional target of YAP in endothelial cells. YAP and ANG-2 was co-localized in endothelial cells of mouse retina and tumor vessels. Reduced expression and overexpression of YAP in HUVECs resulted in a decrease and an increase of ANG-2 expression, respectively. Chromatin immunoprecipitation analysis and luciferase activity assay of ANG-2 promoter indicated that ANG-2 was transcriptionally regulated by YAP. The addition of recombinant ANG-2 protein partially compensated vascular defects caused by YAP depletion. Altogether, these results indicate that ANG-2 transcriptionally regulated by YAP in endothelial cells can promote vascular remodeling and angiogenesis in an autocrine way.

In conclusion, our results suggest that YAP plays significant roles in genetic regulation during vascular remodeling and sprouting (Fig. 1). Angiogenesis starts from endothelial cell activation which accompanies dramatic changes in cell junctions, cytoskeleton, and gene expression. However, it has not been clearly defined on how these events are linked and cooperated. Since abnormal endothelial cell activation occurs in multiple pathophysiological conditions including tumor and inflammation, understanding the molecular mechanism that mediates endothelial cell junctional stability and activation is essential to the development of therapeutic means to improve these pathologic conditions. Although further studies are needed to understand what factors will destabilize endothelial junctions that affect YAP activity and how, our results revealed the role of YAP as a linker connecting endothelial cell junctional stability to genetic changes during vascular remodeling, thus providing further insight into the molecular mechanisms underlying the angiogenic activation of endothelial cells by YAP.

\section{ACKNOWLEDGEMENTS}

This work was supported by grants (NRF-2015R1A2A1A05001859; NRF-2013M3A9B6046563; NRF-2011-0019267 to YGK) from the National Research Foundation of Korea and a grant (A085136 to HJC) from the Korea Health 21 R\&D Project, Ministry of Health and Welfare, Republic of Korea. Authors would like to thank Dong-Su Jang, MFA (Medical Illustrator, Medical Research Support Section, Yonsei University College of Medicine, Seoul, Korea) for his help with the illustrations. 